\title{
A estabilidade do avanço mandibular cirúrgico por meio da osteotomia bilateral sagital: uma revisão
}

Danilo Furquim Siqueira*, Aline Maria Alencar de Castro**, José Antônio dos Santos Júnior**, Liliana Ávila Maltagliati*, Fernanda Angelieri*

\begin{abstract}
Resumo
Introdução: a Osteotomia Sagital Bilateral Mandibular (OSBM) é a cirurgia de escolha da maior parte dos cirurgiões para a correção de discrepâncias esqueléticas mandibulares no sentido sagital. Contudo, sua estabilidade permanece controversa na literatura. Objetivo: neste trabalho, analisaram-se criteriosamente os vários fatores relacionados com a sua estabilidade em longo prazo, como a quantidade de avanço mandibular, o tipo de fixação, a utilização ou não de splints cirúrgicos, o posicionamento condilar, além de outros itens. Conclusão: baseado na literatura consultada, concluiu-se que a magnitude dos movimentos sagitais mandibulares deverá ser menor do que $10 \mathrm{~mm}$; que a fixação rígida deverá ser escolhida em vez da semirígida; que a utilização de splints deverá ser evitada ou feita por tempo reduzido; e que os tratamentos ortodônticos pré e pós-cirúrgicos são essenciais para a obtenção de resultados satisfatórios.
\end{abstract}

Palavras-chave: Avanço mandibular. Estabilidade. Osteotomia sagital.

\section{INTRODUÇÃO}

A Osteotomia Bilateral Sagital Mandibular (OSBM) está indicada para a correção esquelética de pacientes com má oclusão de Classe II com deficiência ou retroposicionamento mandibular, na qual é necessário o avanço mandibular no intuito de harmonizar a estética facial e o relacionamento oclusal. Esta técnica, bastante popular, foi introduzida na década de 50 e sofreu várias modificações ao longo do tempo ${ }^{6}$.

O sucesso da OSBM está diretamente relacionado à estabilidade pós-cirúrgica. A estabilida- de pós-cirúrgica é fundamental na manutenção das alterações faciais e oclusais nos sentidos horizontal e vertical, promovidas pelo novo posicionamento mandibular ${ }^{18,23}$. Entre os fatores que podem influenciar a estabilidade pós-cirúrgica estão: deslocamento do côndilo da cavidade articular após a cirurgia, ação dos músculos e tecidos moles, falta de controle do segmento proximal durante o procedimento cirúrgico, tipos e período de fixação, magnitude do avanço mandibular, crescimento pós-cirúrgico, distúrbios temporomandibulares prévios e idade do paciente no

* Doutores em Ortodontia pela FOB-USP e Professores do Programa de pós-graduação em Odontologia, área de concentração em Ortodontia da Universidade Metodista de São Paulo - UMESP.

** Alunos do programa de pós-graduação em Odontologia, área de concentração em Ortodontia da Universidade Metodista de São Paulo - UMESP. 
momento da cirurgia ${ }^{1,2,6,31}$. Os efeitos decorrentes da recidiva cirúrgica geralmente envolvem movimentos horizontais ou verticais da mandíbula, assim como remodelação ou reabsorção do côndilo mandibular na cavidade articular ${ }^{2}$, sendo que as recidivas mais freqüentes são vistas nos pacientes dolicofaciais ${ }^{24}$.

O objetivo do presente estudo consistiu em revisar a literatura que avalia a estabilidade do avanço mandibular cirúrgico (OBSM), de forma a avaliar a importância do tratamento ortodôntico pré e pós-cirúrgico, fatores verticais e horizontais recidivantes, a utilização pós-cirúrgica de goteiras e/ou elásticos, para a obtenção de um resultado mais estável em longo prazo.

\section{REVISÃO DE LITERATURA E DISCUSSÃO}

A Osteotomia Bilateral Sagital Mandibular (OBSM) é um dos procedimentos cirúrgicos mais utilizados para o avanço mandibular de pacientes adultos com má oclusão de Classe II por deficiência ou retroposicionamento mandibular ${ }^{25}$. Ela pode ser associada a outros procedimentos cirúrgicos, dependendo de cada caso. A cirurgia maxilomandibular combinada é comum em pacientes com retro-posicionamento da mandíbula associado ao excesso vertical e à deficiência ânteroposterior da maxila. Uma mentoplastia também pode ser necessária, no sentido de aumentar ou diminuir o tamanho do mento após o avanço da mandíbula.

Blomqvist e Isaksson ${ }^{8}$ avaliaram a estabilidade da osteotomia sagital mandibular em 38 pacientes, sendo que 11 deles foram submetidos à cirurgia combinada maxilomandibular, 3 à mentoplastia e os outros 24 fizeram só o avanço mandibular. O procedimento cirúrgico, isolado ou combinado, foi considerado estável. De maneira semelhante, outros autores associaram a OSBM à osteotomia maxilar $^{15,22}$ e à mentoplastia ${ }^{21,30}$, relatando estabilidade pós-cirúrgica. Já, quando a OSBM foi comparada à osteotomia subapical mandibular total (OSMT) no período de um ano pós-cirúr- gico, concluiu-se que os procedimentos cirúrgicos variaram de acordo com a indicação, mas ambos apresentaram-se igualmente estáveis na correção da má oclusão de Classe II $^{25}$.

Contudo, existem alguns fatores, relacionados a seguir, que determinam uma maior ou menor estabilidade do avanço mandibular cirúrgico.

\section{Quantidade de avanço mandibular}

Alguns estudos relataram que quanto maior a quantidade de avanço mandibular realizada no procedimento cirúrgico, maior a recidiva ${ }^{1,8,10,11,21,23}$, sendo que um dos fatores relacionados é a adaptação neuromuscular após a cirurgia ${ }^{2,31}$. Por outro lado, Emshoff et al. ${ }^{15}$ não encontraram correlação significante entre a quantidade de avanço mandibular cirúrgico e as alterações horizontais no período pós-cirúrgico.

Todavia, Bailey, Cevidanes e Proffit ${ }^{5}$ afirmaram em seu trabalho que quanto maior o avanço mandibular, maior será sua modificação e, por conseqüência, maior a tendência de recidiva (ou seja, proporcional à alteração). Segundo estes autores, para avanços mandibulares menores que $8 \mathrm{~mm}$, a tendência de recidiva seria significantemente reduzida. O avanço mandibular máximo entre $8 \mathrm{~mm}$ e $10 \mathrm{~mm}$ parece ser consenso entre os trabalhos com a $\mathrm{OSBM}^{23}$. Estes autores criaram uma classificação que resume e define a magnitude da estabilidade pós-cirúrgica em: altamente estável - com menos de $10 \%$ de chance de modificação (ou recidiva) significante pós-tratamento; estável - com menos de $20 \%$ de chance de recidiva pós-tratamento e também nos casos onde esta recidiva ocorre em uma técnica cirúrgica única, na qual a fixação rígida interna (FIR) é comumente utilizada; problemática - com diversas modificações e alterações pós-cirúrgicas. Esse tipo de classificação consegue agrupar a porcentagem dos pacientes operados com OSBM ou com qualquer outro tipo de técnica cirúrgica empregada na mandíbula (Classe II), na qual a estabilidade do avanço ao longo do tempo será analisada. 


\section{Tipos de fixação}

Existem dois tipos básicos de fixação: rígida e semi-rígida. Na fixação semi-rígida são utilizados fios intermaxilares de aço inoxidável. Já a fixação rígida é um procedimento mais moderno e utiliza miniplacas de aço inoxidável ou titânio e vários tipos de parafusos.

Atualmente, a fixação rígida tem sido mais utilizada pelos cirurgiões em relação à semi-rígida ${ }^{4}$, $8,10,11,15,16,20,21,22,25,26,27,28$. Na comparação da fixação rígida com a semi-rígida, Berger et al. ${ }^{6}$ e Watzke et $a 1 .{ }^{30}$ encontraram estabilidade semelhante no período de 1 ano após a cirurgia. Da mesma forma, Dolce et al. ${ }^{13}$ avaliaram a estabilidade 2 anos após a OSBM, quando compararam os dois tipos de fixação, sendo que ambas foram consideradas estáveis. Will e West ${ }^{31}$, analisando uma amostra de 235 pacientes - que, após o ato cirúrgico, receberam fixação óssea semi-rígida seguida da fixação maxilomandibular por 6 semanas - observaram que em $61 \%$ da amostra não houve recidiva no avanço mandibular, contradizendo alguns autores que afirmam ser o avanço mandibular instável e recidivante ${ }^{6,13,14}$.

Na realidade, poucas modificações são verificadas nas posições vertical, horizontal e rotacional da mandíbula durante os primeiros 6 meses após a osteotomia sagital mandibular, quando se utiliza miniplacas monocorticais para a fixação dos segmentos ósseos ${ }^{1}$. Na pesquisa de Borstlap et al. ${ }^{10,11}$, foram utilizadas miniplacas monocorticais em todos os pacientes da amostra, que posteriormente apresentaram $84 \%$ de estabilidade oclusal no período de 2 anos após a cirurgia.

É importante salientar que a fixação rígida pode ser realizada utilizando-se parafusos de fixação dos mais diversos tamanhos. Estes podem ser reabsorvíveis (co-polímeros) ou não-reabsorvíveis (aço inoxidável, titânio, ouro e vitalium). Segundo Ferretti e Reyneke ${ }^{16}$, em seu estudo após 12 meses, as perfurações ósseas dos parafusos reabsorvíveis ainda seriam visíveis (degradação incompleta do co-polímero), porém, após 24 meses, seriam totalmente invisíveis e no local da sua inserção observou-se neoformação óssea. Ainda, segundo estes autores ${ }^{16}$, os parafusos metálicos apresentam uma maior estabilidade em relação aos copolímeros, promovendo uma menor recidiva, contudo sem diferença estatisticamente significante. Porém, Kallela et al. ${ }^{20}$ obtiveram boa estabilidade 1 ano após a cirurgia, quando utilizaram parafusos reabsorvíveis para a fixação rígida. Mathews et al. ${ }^{22}$ encontraram estabilidade semelhante quando comparados os parafusos de titânio e os reabsorvíveis, no período de 6 meses a 1 ano após a osteotomia sagital mandibular.

Blowqvist e Isaksson ${ }^{8}$ compararam a estabilidade pós-cirúrgica, 6 meses após a osteotomia sagital mandibular, quando dois tipos de fixação rígida foram utilizados: parafusos de titânio bicorticais e miniplacas monocorticais. A estabilidade mostrou-se semelhante entre os dois grupos. Contudo, os parafusos metálicos apresentam algumas desvantagens como: podem ser vísíveis intrabucalmente, migrar dentro dos fragmentos ósseos, distorcer imagens de ressonância magnética ou de tomografia computadorizada, além de afetar a palpação na região $0^{22}$.

\section{Tratamento ortodôntico}

O tratamento ortodôntico nas fases pré e póscirúrgicas é de fundamental importância no sucesso do procedimento cirúrgico, assim como na estabilidade pós-cirúrgica ${ }^{20}$. O preparo ortodôntico (Ortodontia pré-cirúrgica) apresenta-se como um ponto crucial no sucesso do tratamento combinado, uma vez que este passo dará todas as condições ideais para que a cirurgia seja bem realizada e, conseqüentemente, aumente a estabilidade do tratamento ${ }^{20}$.

O período de tratamento ortodôntico pós-cirúrgico pode variar de acordo com a qualidade da cirurgia e do tratamento ortodôntico pré-cirúrgico. Este tratamento pode ter início de 4 a 6 semanas após a cirurgia ${ }^{4,8,9,16,27}$ e se estender por aproximadamente 9 meses $^{10,11}$. 
Em uma análise do tempo total de tratamento para a correção da má oclusão de Classe II, a combinação do tratamento ortodôntico fixo com a cirurgia ortognática por meio da OSBM apresentou menor tempo de tratamento, comparado ao grupo tratado com Ortopedia, Ortodontia e distração osteogênica ${ }^{12}$.

\section{Elásticos intermaxilares}

Os elásticos intermaxilares, indicados nos casos onde a fixação rígida foi realizada, deverão ser utilizados no período pós-cirúrgico, de forma contínua, por aproximadamente 3 semanas $^{8}$. Após este período, o uso deverá ser mais flexível, principalmente nos procedimentos de higienização. Deve ser enfatizada a adesão do paciente operado ao protocolo de uso dos elásticos, pois só assim será obtida a estabilidade necessária e o reparo fisiológico no local operado. Elásticos bem apertados devem ser usados entre os primeiros 2 a 5 dias após a cirurgia ${ }^{10,11}$. Após este período, nas próximas 6 semanas, preconiza-se o uso mais relaxado do elástico e estimula-se o paciente a exercitarse com aberturas bucais de até $15 \mathrm{~mm}$, sendo o acompanhamento de um fisioterapeuta necessário e imprescindível. Decorrido este período, reiniciase o tratamento ortodôntico do recém-operado (OSBM).

\section{Goteiras cirúrgicas}

Outro ponto bastante controverso na literatura é a utilização da goteira cirúrgica e/ou de splints no período pós-cirúrgico, com a finalidade de orientação do avanço mandibular. Isto também pode exercer uma influência significativa na estabilidade durante a fase pós-cirúrgica.

Os splints podem ser utilizados por 6 semanas nos pacientes submetidos à fixação semi-rígida (com fios intermaxilares de aço inoxidável) e apenas 5 a 7 dias naqueles nos quais foi realizada a fixação rígida (placas ou parafusos) ${ }^{13,30}$. Contrariamente a isto, Arnett ${ }^{2}$ ressaltou que a goteira (ou splint) deverá ser utilizada cada vez menos em etapas finais cirúrgicas, por influenciar e ocasionar inclinações indesejadas no posicionamento dos côndilos e também por causar rotações indesejadas do plano oclusal.

Os resultados dos estudos sobre a estabilidade pós-cirúrgica deverão ser vistos com cautela, porque muitos deles estão baseados em radiografias feitas imediatamente após o procedimento cirúrgico, com os splints ainda na boca. Com a retirada do splint (para a tomada radiográfica), a mandíbula poderá se deslocar suavemente para frente, fornecendo dados incorretos quando estas radiografias forem comparadas com as posteriores ${ }^{21}$.

\section{Local da recidiva}

Com relação à OBSM, principalmente nos casos de avanço mandibular, a recidiva esquelética pode ser localizada em apenas duas posições anatômicas: no local da osteotomia (deslize entre os segmentos); e na articulação temporomandibular (inclinação condilar ou compressão condilar com alterações morfológicas) $)^{2,3,4}$. O deslize dos segmentos da osteotomia pode ser caracterizado pela redução do comprimento entre o condílio (Co) e os incisivos inferiores, que ocorre no local cirúrgico da OSBM antes da ossificação. Este acontecimento se dá em decorrência do estiramento dos tecidos conjuntivos paramandibulares (TCP), como a pele, o tecido subcutâneo, os músculos e o periósteo, devido ao procedimento cirúrgico, que no período pós-cirúrgico produzem uma força de tração sobre o segmento anteriormente avançado. Neutralizando o vetor TCP está a fixação, mas se o equipamento for ineficaz, a mandíbula se encurta ao longo da osteotomia e ocorre recidiva precoce. Em resposta ao deslize da osteotomia e subseqüente movimento posterior da parte anterior da mandíbula, observam-se compensações dentárias nos incisivos superiores e inferiores, em decorrência da utilização dos elásticos intermaxilares, mascarando recidivas esqueléticas suaves. Estas alterações dentárias poderão recidivar em longo prazo, após a remoção dos elásticos e do apare- 
lho ortodôntico. Durante o ato cirúrgico, pode-se promover o estiramento da musculatura (ventre anterior do digástrico e supra-hióideos) para diminuir a força dos mesmos e conseqüentemente diminuir a tendência de recidiva nos avanços mandibulares $2,3,4$.

A recidiva mandibular na articulação temporomandibular pode ser causada em decorrência de uma "inclinação condilar sem contato", ou seja, quando o côndilo se posiciona inferior ou ântero-inferiormente em relação à posição de acomodação na fossa glenóide e, por causa desta posição, não tem condições de sustentar a mandíbula na posição avançada. Além disto, a recidiva pode ocorrer por uma compressão condilar e uma conseqüente alteração morfológica, devido a um posicionamento inadequado do côndilo no ato cirúrgico ${ }^{2,3,4}$.

Os estudos de Harris, Van Sickels e Alder ${ }^{19}$ mostraram que os côndilos assumem uma posição mais medial, posterior e superior, imediatamente após a cirurgia, em relação à sua posição fisiológica. Na comparação da fixação rígida com a semirígida, a posição final do côndilo foi superior e posterior após a cirurgia de avanço mandibular ${ }^{29}$. Contrariamente, Arnett ${ }^{2}$ ressaltou que, após a OSBM, o côndilo apresenta uma folga em relação à fossa articular. Quando esta folga ocorre sem nenhum contato com as paredes da fossa, o risco de recidiva é maior. O retorno do côndilo à posição original pré-cirúrgica só ocorrerá cerca de 8 semanas após o procedimento cirúrgico. A instabilidade ocorre nestas 8 semanas de ausência de contato condilar com a fossa e sua magnitude vai variar de acordo com o tipo de fixação utilizado. $\mathrm{Na}$ fixação rígida, o contato condilar é maior e, portanto, a estabilidade será maior.

A recidiva por compressão e alteração condilar ocorre por compressão posterior ou medial-lateral durante o procedimento cirúrgico, causando remodelação condilar. Esta remodelação pode ter início entre 9 e 18 meses após a cirurgia ${ }^{2}$. À medida que o côndilo se remodela, ele se reposiciona mais para cima em relação à cavidade articular, causando recidiva mandibular e acentuando a má oclusão de Classe II.

A remodelação condilar idiopática ${ }^{2,3,4}$ pode ser responsável pela recidiva mandibular e pode ocorrer em adultos e em jovens. Arnett et al. 2,3,4 acreditam que o fator genético seria o principal responsável pela diminuição no comprimento mandibular (em adultos) e por uma redução na taxa de crescimento deste osso naqueles ainda em crescimento, seguido por fatores ambientais (alergias, hábitos, etc).

Bettega et al. ${ }^{7}$ definiram que os côndilos deveriam ficar o mais próximo da posição anterior no ato operatório e que há uma forte relação entre a estabilidade em longo prazo e a parte óssea avançada.

Por outro lado, a associação da osteotomia (OSBM) e as disfunções temporomandibulares (DTM) ainda é obscura e controversa. Alguns autores ${ }^{2,10,11,19}$ afirmaram que pacientes com DTM pré-cirúrgica apresentaram melhora nos sinais e sintomas após a cirurgia e alguns, que não demonstravam distúrbios articulares antes da cirurgia, apresentaram disfunções no período pós-cirúrgico. Não houve influência de idade ou gênero na prevalência de distúrbios temporomandibulares ${ }^{10,11}$. $\mathrm{Na}$ verdade, naqueles pacientes com DTM e má oclusão de Classe II, existe uma grande dificuldade de posicionar o côndilo corretamente na fossa articular durante a cirurgia ortognática ${ }^{17}$.

Existem quatro métodos de fixação e posicionamento dos côndilos descritos por Bettega et al. ${ }^{7}$ : 1) método empírico, no qual a experiência do cirurgião é que ditará a posição condilar; 2) método no qual pontos anatômicos e dentários são objetos de referência no posicionamento do côndilo; 3) Método radiológico ou que utilize outras técnicas mais recentes para posicionamento dos côndilos; 4) método no qual os côndilos são posicionados por dispositivos modernos. $\mathrm{Na}$ maioria das vezes, esses dispositivos são incômodos e de difícil manuseio por parte do operador, demandando 
tempo de cirurgia e sendo visivelmente desvantajosos.

No futuro, em cirurgias ortognáticas como a OSBM, acredita-se que a utilização da Robótica será fundamental para o posicionamento dos côndilos. Fatores individuais, inerentes a cada cirurgia e à técnica cirúrgica empregada, determinarão elementos que serão levados em conta junto à fixação utilizada, o uso correto de contenção pelo período determinado e, mais importante, o respeito pela geometria mandibular e o plano oclusal.

\section{CONCLUSÃO}

Baseado na literatura consultada, conclui-se que, para a obtenção de uma estabilidade satisfatória, alguns requisitos devem ser observados:
- os avanços mandibulares maiores do que $10 \mathrm{~mm}$ devem ser evitados;

- a fixação rígida deve ser escolhida em vez da semi-rígida;

- a utilização de goteiras e splints pós-cirúrgicos deve ser evitada ou feita por tempo reduzido;

- os elásticos intermaxilares devem ser utilizados no período pós-cirúrgico, de acordo com a recomendação do cirurgião;

- os tratamentos ortodônticos pré e pós-cirúrgicos são essenciais para a obtenção do sucesso do tratamento ortodôntico-cirúrgico.

\title{
Stability of surgical mandibular advancement using bilateral sagittal split osteotomy: a review
}

\begin{abstract}
Introduction: The bilateral sagittal split osteotomy (BSSO) is preferred by most surgeons to correct mandibular sagittal skeletal discrepancies. However, studies on its stability are controversial. Aim: This study evaluated the different factors associated with long-term stability, such as quantity of mandibular advancement, type of fixation, utilization or not of surgical splints, condylar position, and others. Conclusion: Based on the literature, it was concluded that the magnitude of mandibular sagittal movement should be less than $10 \mathrm{~mm}$; rigid fixation should be chosen instead of semi-rigid; the utilization of splints should be avoided or used for a short period; and pre- and postsurgical orthodontic treatments are essential to obtain satisfactory results.
\end{abstract}

Key words: Mandibular advancement. Stability. Sagittal osteotomy.

\section{REFERÊNCIAS}

1. ABELOOS, J.; DE CLERCQ, C.; NEYT, L. Skeletal stability following miniplate fixation after bilateral sagittal split osteotomy for mandibular advancement. J. Oral Maxillofac. Surg. Philadelphia, v. 51, p. 366-369, Apr. 1993.

2. ARNETT, G. W. A redefinition of bilateral sagittal osteotomy (BSO) advancement relapse. Am. J. Orthod. Dentofacial Orthop., St. Louis, v. 104, no. 5, p. 506-515, Nov. 1993.

3. ARNETT, G. W.; MILAM, S. B.; GOTTESMAN, L. Progressive mandibular retrusion - idiopathic condilar resorption. Part I. Am. J. Orthod. Dentofacial Orthop., St. Louis, v. 110, no. 1, p. 8-15, July 1996.

4. ARNETT, G. W.; MILAM, S. B.; GOTTESMAN, L. Progressive mandibular retrusion -idiopathic condilar resorption. Part II. Am. J. Orthod. Dentofacial Orthop., St. Louis, v. 110, n. 2, p. 117-127, Aug. 1996.
5. BAILEY, L. T. J.; CEVIDANES, L. H.; PROFFIT, W. R. Stability and predictability of orthognathic surgery. Am. J. Orthod. Dentofacial Orthop., St. Louis, v. 126, no. 3, p. 273-277, Sept. 2004.

6. BERGER, J. L.; PANGRAZIO-KULLBERSH, V.; BACCHUS, S. N.; KACZYNSKI, R. Stability of bilateral sagittal split ramus osteotomy: rigid fixation versus transosseous wiring. Am. J. Orthod. Dentofacial Orthop., St. Louis, v. 118, no. 4, p. 397-403, Oct. 2000.

7. BETTEGA, G.; DESSENNE, V.; RAPHAËL, B.; CINQUIN, P. Computer-assisted mandibular condyle posicioning in orthognathic surgery. J. Oral Maxillofac. Surg., Philadelphia, v. 56, p. 553-558, May 1996.

8. BLOMQVIST, J. E.; ISAKSSON, S. Skeletal stability after mandibular advancement: a comparison of two rigid internal fixation techniques. J. Oral Maxillofac. Surg., Philadelphia, v. 52, p. 1133-1137, Nov. 1994. 
9. BLOMOVIST, J. E.: AHLBORG, G.; ISAKSSON, S.; SVARTZ, K. A comparison of skeletal stability after mandibular advancement and use of two rigid internal fixation techniques. J. Oral Maxillofac. Surg., Philadelphia, v. 55, p. 568-574, June 1997

10. BORSTLAP, P. J.; STOELINGA, P. J. W.; HOPPENREIJS, T. J. M.; VAN'T HOF, M. A. Stabilization of sagittal split advancements osteotomies with miniplates: a prospective, multicentre study with two-year follow-up. Part I: clinical parameters. Int. J. Oral Maxillofac. Surg., Copenhagen, v. 33, no. 5, p. 433-441, July 2004.

11. BORSTLAP, P. J.; STOELINGA, P. J. W.; HOPPENREIJS, T. J. M.; VAN'T HOF, M. A. Stabilization of sagittal split advancements osteotomies with miniplates: a prospective, multicentre study with two-year follow-up. Part II. Radiographic parameters. Int. J. Oral Maxillofac. Surg., Copenhagen, v. 33, no. 6, p. 535542, Sept. 2004

12. BREUNING, H. K.; VAN STRIJEN, J. P.; PRAHL-ANDERSEN, B.: TUINZING, B. D. Duration of orthodontic treatment and mandibular lengthening by means of distraction or bilateral split osteotomy in patiens with Angle Class II malocclusions. Am. J. Orthod. Dentofacial Orthop., St. Louis, v. 127, no. 1, p. 25-29, Jan. 2005

13. DOLCE, C.; VAN SICKELS, J. E.; BAYS, R. A.; RUGH, J. D. Skeletal stability after mandibular advancement with rigid versus wire fixation. J. Oral Maxillofac. Surg., Philadelphia, v. 58 no. 11, p. 1219-1227, Nov. 2000

14. DOLCE, C.; HATCH, J. P.; VAN SICKELS, J. E.; RUGH, J. D. Rigid versus wire fixation for mandibular advancement: skeletal and dental changes after 5 years. Am. J. Orthod. Dentofacial Orthop., St. Louis, v. 121, no. 6, p. 610-619, June 2002.

15. EMSHOFF, R.; SCHEIDERBAUER, A.; GERHARD, S.; NORER, B Stability after rigid fixation of simultaneous maxillary impactation and mandibular advancement osteotomies. Int. J. Oral Maxillofac. Surg., Copenhagen, v. 32, no. 2, p. 137-142, Apr. 2003.

16. FERRETTI, C.; REYNEKE, J. P. Mandibular sagittal split osteotomies fixed with biodegradable or titanium screws: a prospective, comparative study of postoperative stability. Oral Surg. Oral Med. Oral Pathol. Oral Radiol. Endod., St. Louis, v. 93, no. 5, p. 534-537, May 2002

17. GAGGL, A.; SCHULTES, G.; SANTKER, G.; KARCHER, H. SIMBRUNNER, J. Clinical and magnetic resonance findings in the temporomandibular joints of pacients before and after orthognatic surgery. Br. J. Oral Maxillofac. Surg., Edinburgh, v. 37 , no. 1, p. 41-45, Feb. 1999.

18. GREEBE, R. B.; TUINZING, D. B. Mandibular advancement procedures: predictable stability and relapse. Oral Surg. Oral Med. Oral Pathol., St. Louis, v. 1, no. 57, p. 13-16, Jan. 1984.

19. HARRIS, M. D.; VAN SICKELS, J. E.; ALDER, M. Factors influencing condilar position after the bilateral sagittal split osteotomy fixed with bicortical screws. J. Oral Maxillofac. Surg. Philadelphia, v. 57, no. 6, p. 650-654, June 1999
20. KALLELA, I.: LAINE, P. SUURONEN, R. IIZUKA, T: PIRINEN, S.; LINDQVIST, C. Skeletal stability following mandibular advancement and rigid fixation with polylactide biodegradable screws. Int. J. Oral Maxillofac. Surg., Copenhagen, v. 27 no. 1, p. 3-8, Feb. 1998.

21. KIERL, M. J.; NANDA, R. S.; CURRIER, G. F. A 3-year evaluation of skeletal stability of mandibular advancement with rigid fixation. J. Oral Maxillofac. Surg., Philadelphia, v. 48, no. 6 , p. 587-592, June 1990

22. MATTHEWS, N. S.; KHAMBAY, B. S.; AYOUB, A. F.; KOPPEL, D.; WOOD, G. Preliminary assessment of skeletal stability after sagittal split mandibular advancement using a bioresorbable fixation system. Br. J. Oral Maxillofac. Surg., Edinburgh, v. 41, no. 3, p. 179-184, June 2003

23. MCDONALD, W. R. Stability of mandibular lengthening: a comparison of moderate and large advancements. J. Oral Maxillofac. Surg., Philadelphia, v. 2, p. 729-735, 1990.

24. MOBARAK, K. A.; ESPELAND, L.; KROGSTAD, O.; LYBERG, T. Mandibular advancement surgery in high-angle and low-angle Class II patients: different long-term skeletal responses. Am. J. Orthod. Dentofacial Orthop., St. Louis, v. 119, no. 4, p. 368381, Apr. 2001.

25. PANGRAZIO-KULBERSH, V.; BERGER, J. L.; KACZYNSKI, R.; SCHUNOCK, M. Stability of skeletal Class II correction with 2 surgical techniques: the sagittal split ramus osteotomy and the total mandibular subapical alveolar osteotomy. Am. J. Orthod. Dentofacial Orthop., St. Louis, v. 120, no. 2, p. 134-143, Aug. 2001

26. PERROTT, D. H.; POGREL, M. A.; KABAN, L. B. Stability of sagittal split osteotomias: a comparison of three stabilization techniques. Oral Surg. Oral Med. Oral Pathol., St. Louis, v. 78, no. 6, p. 696-704, Dec. 1994

27. SCHEERLINCK, J. P.; STOELINGA, P. J.; BLIJDORP, P. A.; BROUNS, J. J. A.; NIJS, M. L. L. Sagittal split advancement osteotomies stabilized with miniplates. A 2-5-year follow-up. Int. J. Oral Maxillofac. Surg., Copenhagen, v. 23, no. 3, p. 127-131, June 1994

28. STOELINGA, P. J. W.; BORSTLAP, W. A. The fixation of sagittal split osteotomies with miniplates: the versatility of a technique. J. Oral Maxillofac. Surg., Philadelphia, v. 61, no. 12, p. 14711476, Dec. 2003

29. VAN SICKELS, J. E.; TINER, B. D.; KEELING, S. D.; CLARK, G. M.; BAYS, R.; RUGH, J. Condylar position with rigid fixation versus wire osteosynthesis of a sagittal split advancement. J. Oral Maxillofac. Surg., Philadelphia, v. 57, no. 1, p. 31-34, Jan. 1999

30. WATZKE, I. M.; TURVEY, T. A.; PHILLIPS, C.; PROFFIT, W. R. Stability of mandibular advancement after sagittal osteotomy with screw or wire fixation: a comparative study. J. Oral Maxillofac. Surg., Philadelphia, v. 48, no. 2, p. 108-121, Feb. 1990.

31. WILL, L. A.; WEST, R. A. Factors influencing the stability of the sagittal split osteotomy for mandibular advancement. J. Oral Maxillofac. Surg., Philadelphia, v. 47, p. 813-818, 1989.
Endereço para correspondência

Danilo Furquim Siqueira

Rua Costa Aguiar, 875, Apto 111,

CEP: 04.204-000 - Ipiranga, São Paulo/SP

E-mail: danilofurquim@uol.com.br 